\title{
Electromyographic and skin resistance responses to osteopathic manipulative treatment for low-back pain
}

\author{
STEPHEN M. ELLESTAD, DO \\ ROBERT V. NAGLE, DO \\ DAVID R. BOESLER, DO \\ MEARL A. KILMORE, PHD
}

Previous studies have demonstrated that patients with low-back pain have increases in absolute electromyographic (EMG) activity levels in the lumbar paraspinal muscles during motion. In the present study, EMG, skin resistance, and supine lumbar lordosis measurements in patients with low-back somatic dysfunction were evaluated. The 40 subjects in the study ranged in age from 22 to 36 years. Twenty had low-back pain and 20 were pain-free (controls). In each group, 10 subjects were assigned randomly to receive osteopathic manipulative treatment (OMT), which consisted of high-velocity, low-amplitude techniques for the entire axial skeleton and pelvis. The OMT subjects had measurements taken before and after treatment, while the control group was measured before and after a period of rest. All subjects were re-evaluated seven days later. There were significant decreases in EMG activity during motion and in skin resistance levels in the low-back pain treatment group, as well as a significant decrease in EMG levels in the control OMT group. No significant differences were found in the low-back pain and control nontreatment groups.

Many low-back pain sufferers consult an osteopathic physician because of the general perception that osteopathic manipulative treatment (OMT) can reduce the severity of such pain. ${ }^{1}$ However, there is a scarcity of objective data to support this concept.

A major problem in evaluating low-back pain treatment programs is the lack of accepted objective criteria or procedures to assess therapeutic progress. ${ }^{2}$ Some investigators ${ }^{3-5}$ have used the surface electromyogram (EMG) to aid in ascertaining both the severity of the pain and the ability of treatment to relieve it.

The objectives of the present study were to determine whether evaluation of the effectiveness of OMT in low-back pain can be based on the EMG, and whether quantitative parameters relative to OMT's efficacy could be found. Because skin resistance has been associated with stress-tension levels and OMT has been correlated with relieving tension in patients, ${ }^{6}$ resistance measurements were made. Also, lumbar lordosis was measured to determine whether the low-back pain was associated with an exaggerated lordotic curve that could be relieved or decreased with OMT.

\section{Methods}

\section{Subject selection}

The 26 men and 14 women (age range, 22 to 36 years) in this study were selected from three populations: potential patients of the OMT department; first-year students of the university; and spouses or friends of university students.

Twenty of the 40 subjects complained of lowback pain of at least two weeks' but no longer than six months' duration. The area of pain or spasm 
varied among subjects, but it was localized either unilaterally or bilaterally in the erector spinae musculature between L-2 and S-1. Ten subjects were assigned randomly (alternately) to the OMT group, while the other 10 were assigned to the control group.

The other 20 subjects denied any history of lowback pain during the six months prior to the study. Deep palpation of these persons failed to elicit pain in the lumbosacral area. The same alternating randomization procedure was used for these subjects. All 40 subjects had not received previous OMT for musculoskeletal problems. All evaluations and treatments were performed by one of three Osteopathic Manipulative Medicine (OMM) fellows.

All subjects were informed of possible risks and benefits of the study, and all signed the informed consent form approved by the Health, Safety and Human Use Committee of University of Osteopathic Medicine and Health Sciences-College of Osteopathic Medicine and Surgery (UOMHSCOMS).

The room temperature was maintained between $70^{\circ} \mathrm{F}$ and $74^{\circ} \mathrm{F}$ for all studies.

\section{General procedures}

Physical examination, including sensory, motor, and reflex testing of the lower extremities, was performed for all subjects. Every subject also underwent structural examination (palpation of spine, motor testing), with concentration on areas of the common compensatory pattern (CCP). No low-back pain subject exhibited any neurologic deficit.

All subjects with pain who were not to receive OMT were prescribed a combination of chlorzoxazone and acetaminophen (Parafon Forte); the dosage was two tablets four times a day for 5 days. However, there was no way to verify compliance. Those with pain who were to receive OMT also were given Parafon Forte and instructed to take two tablets four times a day if they continued to have severe pain.

\section{Lumbar lordosis measurements}

Lumbar lordosis measurements were obtained with the subjects supine on a specially designed table (Fig 1). (Various tests documenting the reliability of the table and measurement procedure had been performed previously. ${ }^{7}$ ) The spinous processes of L-1 to L-5 were marked, and then the subject would lie supine on the table. The measurements were recorded in centimeters from each spinous process to the level of the table top. Measurements were repeated five times for each spinous process.

Between complete lumbar spinous process read- ings, the subject assumed a sitting position for five seconds. This was done to obtain consistent results and to relax the spinal musculature, which prevented any lumbar hyperextension from prolonged lying on a hard surface. The mean of the five readings for each spinous process was calculated.

\section{EMG and skin resistance measurement procedures}

An eight-channel Grass polygraph (model 7) with a 7P3 preamplifier was used for direct recording of the surface EMG. In addition, an integrated EMG readout (Fig 2) was obtained simultaneously for each EMG by taking the integrated signal from the preamplifier and feeding it into the driver amplifier of another channel. Skin resistance was recorded on the polygraph using the GSR circuit of a 7P1 preamplifier.

The initial step in recording the surface EMG was to clean the subject's lumbar area with isopropyl alcohol and acetone and allow it to dry. The area was painted with tincture of benzoin to assist in maintaining surface electrode placement. An electrode paste was applied to the surface of each electrode.

Skin resistance electrodes were placed $2 \mathrm{~cm}$ laterally on each side of the spinous process of L-2. The active EMG electrodes were placed $2 \mathrm{~cm}$ laterally from the spinous processes of both L-3 and L5. The ground EMG electrode was placed $4 \mathrm{~cm}$ from the spinous process of L-4. Two sets of EMG electrodes were used (one on each side of the spinous process).

The subject was asked to assume a prone position on a treatment table. After relaxing for five minutes so that EMG potentials formed a flat line, the subject was instructed to bend backward (active lumbar extension), with hands under the chin. This position was held for five seconds. Then the subject assumed a standing position and, after the quiescent period, bent forward until the fingertips passed the inferior borders of the patellas. This position was held for five seconds, and then the subjects returned to an erect standing posture. Next, the subject returned to a prone position on the treatment table, and the same sequence of events was repeated.

\section{OMT technique}

After the second series of recordings, the OMT subjects were then evaluated and treated using the following techniques:

(1) The long restrictors of the hip (hamstrings, hip flexors, and external rotators) were treated with isometric and isotonic muscle energy procedures. 


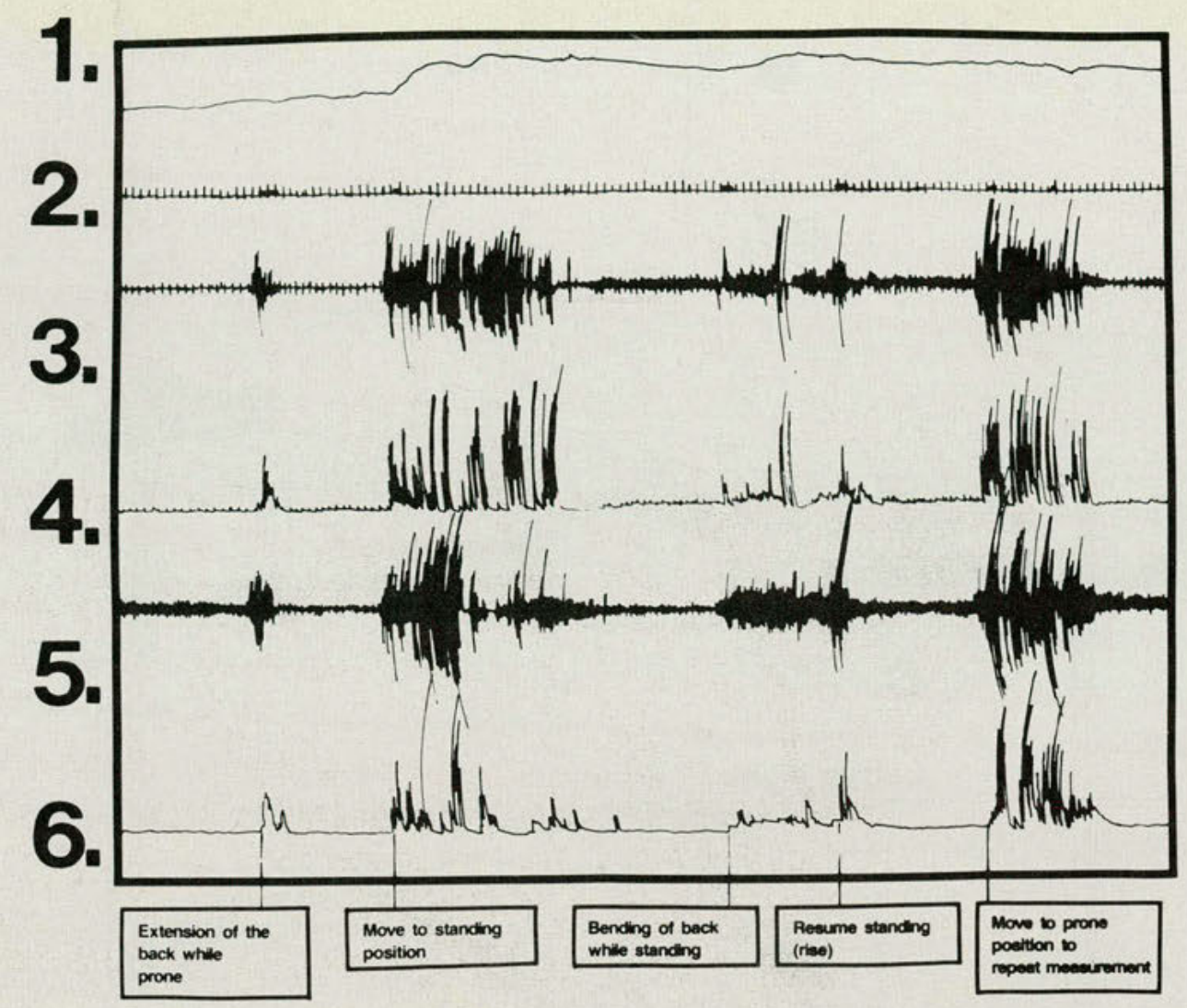

Fig 2. Polygraph tracing show the different parameters recorded and the movements that generated muscular activity, as follows: (1) skin resistance; (2) time (seconds); (3) activity produced by the right lumbar erection spinae musculature; (4) electric summation (integration) of the activity in (3); (5) activity produced by the left lumbar erection spinae musculature; and (6) electric summation (integration) of the EMG activity in (5).

did the preparation. All polygraph record analysis was performed by one of two investigators, who worked together in obtaining the numeric values, which maintained uniformity in this process. Statistical evaluation included the unpaired $t$-test and a two-way analysis of variance.

\section{Results}

The results of skin resistance testing are listed in Table 1 . The individual values were obtained by subtracting the initial value after an equilibration period from the value taken seven days following OMT. Skin resistance was found to be statistically lower when OMT was used for low-back pain.

EMG activity results are shown in Table 2. To obtain these values, the integrated recording of the EMG was used for the active lumbar extension (backward bending) in prone and standing posi- tions. Lumbar extension was chosen as the movement that was most frequently preceded by a quiescent period. Also, it is the primary action that is produced by the lumbar erector spinae musculature. To obtain these values, the initial peak activity of the integrated EMG (obtained by measuring the distance from a baseline to the maximum height) was averaged for the two readings prior to OMT and subtracted from the initial averaged value. The results are consistent in showing that OMT is effective in reducing EMG activity irrespective of the presence of back pain. However, the group with back pain had a greater reduction of EMG activity.

The results of lumbar lordosis measurement are presented in Table 3 . These values were obtained by averaging the readings of L- 1 through L- 5 before the first OMT and subtracting these values 
from comparable values obtained after the second OMT session seven days later. A statistical decrease in lumbar lordosis following OMT was not observed.

All subjects who received OMT reported feeling better following treatment. Both the OMM fellows and the subjects noticed improved range of motion. The low-back pain subjects reported less discomfort following OMT.

\section{Discussion}

A major objective for this investigation was to determine whether OMT could alter measurable values that might coincide with relief of low-back pain.

First, OMT, which used HVLA axial skeleton techniques, decreased EMG activity. This might be related to improved circulation to the injured area, which facilitated tissue repair and reduced the tissue content of mediators that produce pain. The patient does not have to exert as intense an effort to perform the same movement as when pain is present. Improving the range of motion can also be a component for reduced EMG activity after OMT for the nonpain treatment group.

Our subjects with decreased EMG activity postOMT affirmed that it required less effort to perform the study's standard motion. The greater effort was reflected as more electric activity from the muscles when the motion was performed. Abolishment of pain allowed the subjects to be more free in their movements and, thus, to produce less EMG activity.

All patients with low-back pain who received OMT noted a subjective decrease in the intensity of their pain. This was not reported by patients with pain who did not recieve OMT.
Second, the decrease in skin resistance following OMT may be associated with a variation in activity of the sympathetic nerves, as shown by Korr and associates. ${ }^{8}$ Decreased skin resistance also could coincide with a greater degree of relaxation, which frequently was noted after OMT, as well as with diminished anxiety because of less pain.

Less tension within the body and less sympathetic activity can lead to decreased peripheral vascular resistance, which in turn results in better blood flow to the skin and, possibly, to the areas of intense pain. If this occurs, there should be a more rapid return of an injured area to its preinjured condition.

We conclude that the quantitative parameters of skin resistance and EMG activity decreases following OMT are correlated to qualitative responses of less discomfort, improved range of motion, and less tenseness.

Third, the inability to demonstrate that OMT statistically decreased the lumbar lordortic curve was a major disappointment. It was theorized that the spasm of muscles in the back would exaggerate the lordotic curve. If the spasm could be relieved, there should be decreased lumbar lordosis.

Statistical significance may have been obtained had there been more subjects, because the values did tend to reflect a flattening of the lordotic curve, and the statistics tended to approach significance. If the study had been continued for a longer period of time (for example, 1 to 3 months), a more consistent change also may have been seen.

A major way to achieve reproducible results is to evaluate the factors that produce back pain for each patient and to use techniques to influence them. We believe that EMG and skin resistance

\begin{tabular}{|c|c|c|c|c|c|}
\hline \multicolumn{6}{|c|}{ Unpaired $t$-test } \\
\hline & Non-OMT & OMT & & $t$-value & $P$ value \\
\hline $\begin{array}{l}\text { Back pain group } \\
\text { SD }{ }^{\dagger} \\
\text { Non-pain group } \\
\text { SD } \\
t \text {-value } \\
P \text { value }\end{array}$ & $\begin{array}{r}-6,790 \\
\pm 27,090 \\
-14,940 \\
\pm 26,420 \\
0.681 \\
\text { NS }\end{array}$ & $\begin{array}{r}-77,670 \\
\pm 41,210 \\
-20,480 \\
\pm 43,930 \\
3.003 \\
<0.01\end{array}$ & & $\begin{array}{l}4.545 \\
0.342\end{array}$ & $\begin{array}{l}<0.001 \\
\mathrm{NS}^{+}\end{array}$ \\
\hline \multicolumn{6}{|c|}{ Two-way analysis of variance } \\
\hline $\begin{array}{l}\text { Parameter } \\
\text { tested }\end{array}$ & $\begin{array}{l}\text { Sum of } \\
\text { squares }\end{array}$ & $\begin{array}{l}\text { Degrees of } \\
\text { freedom }\end{array}$ & $\begin{array}{l}\text { Mean } \\
\text { square }\end{array}$ & $\begin{array}{c}F \\
\text { value }\end{array}$ & $\begin{array}{c}P \\
\text { value }\end{array}$ \\
\hline Back pain $v$ no pain & 146.000 & 1 & 146.000 & 11.542 & 0.002 \\
\hline OMT $v$ no OMT & 60.123 & 1 & 60.123 & 4.753 & 0.033 \\
\hline \multicolumn{6}{|l|}{ Back pain and OMT } \\
\hline interaction & 106.733 & 1 & 106.733 & 8.438 & 0.006 \\
\hline Error & 455.359 & 36 & 12.649 & & \\
\hline
\end{tabular}




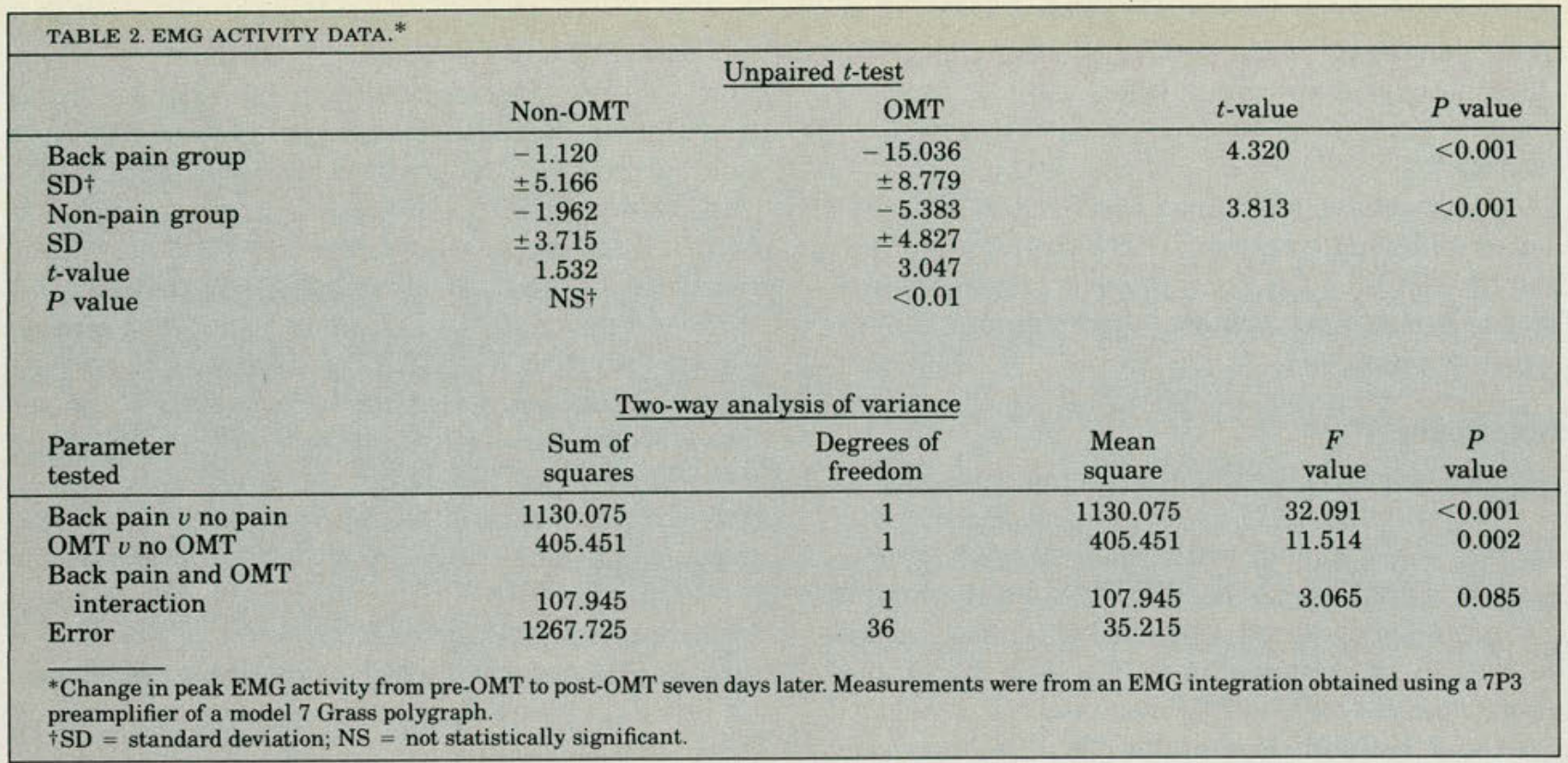

\begin{tabular}{|c|c|c|c|c|c|}
\hline \multicolumn{6}{|c|}{$\underline{\text { Unpaired } t \text {-test }}$} \\
\hline & Non-OMT & OMT & & $t$-value & $P$ value \\
\hline $\begin{array}{l}\text { Back pain group } \\
\text { SD } t \\
\text { Non-pain group } \\
\text { SD } \\
t \text {-value } \\
P \text { value }\end{array}$ & $\begin{array}{r}-0.260 \\
\pm 1.451 \\
-0.628 \\
\pm 2.220 \\
0.439 \\
\text { NS }\end{array}$ & $\begin{array}{r}-0.754 \\
\pm 1.098 \\
-1.574 \\
\pm 1.777 \\
1.242 \\
\text { NS }\end{array}$ & & $\begin{array}{l}0.859 \\
1.052\end{array}$ & $\begin{array}{l}\mathrm{NS}+ \\
\mathrm{NS}\end{array}$ \\
\hline \multicolumn{6}{|c|}{ Two-way analysis of variance } \\
\hline $\begin{array}{l}\text { Parameter } \\
\text { tested }\end{array}$ & $\begin{array}{l}\text { Sum of } \\
\text { squares }\end{array}$ & $\begin{array}{l}\text { Degrees of } \\
\text { freedom }\end{array}$ & $\begin{array}{l}\text { Mean } \\
\text { square }\end{array}$ & $\begin{array}{c}F \\
\text { value }\end{array}$ & $\begin{array}{c}P \\
\text { value }\end{array}$ \\
\hline Back pain $v$ no pain & 5.184 & 1 & 5.184 & 1.820 & 0.182 \\
\hline OMT $v$ no OMT & 3.528 & 1 & 3.528 & 1.238 & 0.272 \\
\hline \multicolumn{6}{|l|}{ Back pain and OMT } \\
\hline interaction & 0.511 & 1 & 0.511 & 0.179 & \\
\hline Error & 102.564 & 36 & 2.849 & & \\
\hline
\end{tabular}

can be used to evaluate therapeutic modalities for our future studies. The two major problems with the EMG as a routine monitoring technique of lowback pain improvement in a normal clinical setting are the expensive equipment needed and the time required for this additional evaluation. However, this technique will be helpful, in the initial evaluation of therapeutic modalities and the value of other procedures.

Our study also demonstrated that more than $90 \%$ of both the control OMT and pain treatment groups met the criteria of the CCP, ${ }^{9}$ as taught by the faculty of the Osteopathic Manipulative Medicine Department at UOMHS-COMS. We have found CCP to be an extremely useful tool in structural diagnosis. An orderly approach to muscular skeletal problems is achieved by following the pattern of CCP concentrates on transitional areas of the spine, which usually shows the greatest ranges and restrictions of motion. Ten percent of the treatment subjects did not fit the pattern in one or two areas; they were treated accordingly.

The basic outline of CCP is as follows:

(1) Upper cervical complex (C-1 and C-2)—rotation left and sidebending right, with occasional left sideslipping and a right anterior attitude of the occiput on the atlas;

(2) thoracic inlet (T-1 to T-4, ribs one and two, 
manubrium, angle of Louis, and both clavicles) rotation right and sidebending right;

(3) thoracic outlet (lower six ribs bilaterally, L1 to L-3, and xiphoid process)_rotation left and sidebending left;

(4) lumbosacral complex (L-4 to S-1)-rotation right and sidebending left;

(5) sacrum-left on left sacral torsion;

(6) innominates-left posterior, right anterior, and outflared;

(7) pubic symphysis-left superior and right inferior; and

(8) long restrictors of the hip-right hamstring contracture, left psoas contracture, and right piriformis contracture.

\section{Comment}

It is our hope that development of protocols such as those described in this report can be useful in the assessment of other osteopathic manipulative methods, including muscle energy, counterstrain, and functional techniques.

This study does establish the effectiveness of high-velocity, low-amplitude OMT for symptomatic and asymptomatic individuals, as measured by absolute EMG potentials and skin resistance in the lumbar area.

This study was supported by a National Osteopathic Foundation special projects grant.

Bernard A. TePoorten, DO, FAAO, and the Division of Osteopathic Medicine at UOMHS-COMS provided training and a re- view of the osteopathic principles and techniques in this study. Wayne H. Terry, PhD, assisted in the development of techniques and the analysis of data.

1. Sims WH, Jayson MIV, Young SMS, et al: Continued trial of mobilization and manipulation for low-back pain in the general practice. $\mathrm{Br}$ Med $J$ 1978;2:1338-1340.

2. Bennett W, et al: Low-back pain: What about chiropractors? Harvard Med School Health Letter January 1988;13:1-8.

3. Hoyt WH, Hunt HH, DePauw MA, et al: Electromyographic assessment of chronic low-back pain syndrome. JAOA 1981;80:728-730.

4. Sherman A: Relationships between strength of low-back muscle contraction and reported intensity of chronic low-back pain. Am J Phys Med 1985;64:190-200

5. Kravitz E, Moore ME, Glaros A.: Paralumbar muscle activity in chronic low-back pain. Arch Phys Med Rehab 1981;62:172-175.

6. Korr IM: Experimental alterations in segmental sympathetic (sweat gland) activity through myofascial and postural disturbances. Fed Proc $1949 ; 8: 88$.

7. Ahsan AM, Jackson TS, Zink JG, et al: A table to measure osteopathic manipulative therapy-induced changes in lumbar lordosis. JAOA $1981 ; 80: 753$

8. Korr IM, Wright HM, Chase JA: Cutaneous patterns of sympathetic activity in clinical abnormalities of the musculoskeletal system. Acta Neuroveg 1964;25:589-606.

9. Zink JG, Lawson WB: An osteopathic structural examination and functional interpretation of the soma. Osteopath Ann 1979;7:12-19.

From the Department of Osteopathic Manipulative Medicine, University of Osteopathic Medicine and Health SciencesCollege of Osteopathic Medicine and Surgery, Des Moines, Iowa.

Reprint requests to Dr Kilmore, University of Osteopathic Medicine and Health Sciences-College of Osteopathic Medicine, Department of Physiology and Pharmacology, 3200 Grand Ave, Des Moines, IA 50312. 